\title{
A SIMPLE TECHNIQUE FOR THE DEMONSTRATION OF URINARY CASTS
}

\author{
BY \\ DOUGLAS B. BREWER \\ From the Department of Experimental Pathology, University of Birmingham
}

(RECEIVED FOR PUBLICATION MARCH 27, 1953)

During recent investigations into the nature of hyaline casts we became impressed again by the well-known difficulty of detecting them under ordinary conditions of microscopic observation and illumination. They can, of course, be rendered more obvious by racking down the substage condenser or by using phase-contrast microscopy, but both these techniques require a certain amount of judgment and do not yield anything like as striking and obvious a result as the method described here. Moreover, this method readily reveals the presence of small numbers of casts and they are easily seen in a rapid survey of the whole area of the coverslip with the lowest power of the microscope.

The method consists of negative staining of the deposit with indian ink. One drop of the centrifuged urinary deposit is mixed with one drop of indian ink (Mandarin Black, Winsor and Newton) on a slide. A coverslip is placed on the mixture and pressed firmly down on to the slide. The excess fluid is wiped from the edges of the coverslip and the slide examined under the microscope. Any casts present are immediately obvious as long, thin white rectangles, standing out against a black background (Fig. 1). Epithelial cells can also be seen, though, for cytological and other purposes, it is better to examine the deposit in the normal way by mounting a drop of urine under a separate coverslip on the same slide.

Examined in this way hyaline casts can be seen in many instances to have long, thin, tapering tails. The swelling and gradual solution of casts in alkaline solution is also well seen.

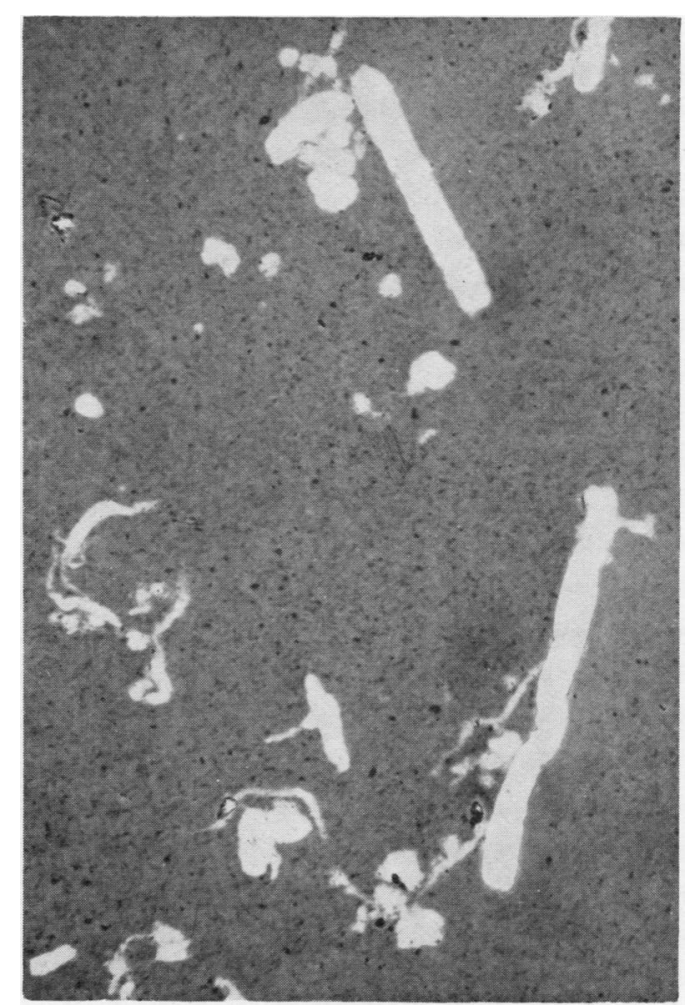

FIo 1.-Urinary deposit containing hyaline casts negatively stained with indian ink, $\times 91$. 